\title{
High-Throughput Sequencing of MicroRNAs in Adenovirus Type 3 Infected Human Laryngeal Epithelial Cells
}

\author{
Yuhua Qi, ${ }^{1,2}$ Jing Tu,, ${ }^{1}$ Lunbiao Cui, ${ }^{2}$ Xiling Guo, ${ }^{2}$ Zhiyang Shi, ${ }^{2}$ Shuchun Li, ${ }^{1}$ Wenting Shi, ${ }^{1}$ \\ Yunfeng Shan, ${ }^{2}$ Yiyue Ge, ${ }^{2}$ Jun Shan,, ${ }^{2}$ Hua Wang, ${ }^{2}$ and Zuhong Lu ${ }^{1}$ \\ ${ }^{1}$ State Key Laboratory of Bioelectronics, School of Biological Science and Medical Engineering, Southeast University, SiPaiLou2\#, \\ Nanjing 210096, China \\ ${ }^{2}$ Key Laboratory of Enteric Pathogenic Microbiology, Ministry of Health, Microbiological Laboratory, Jiangsu Center for Disease \\ Prevention and Control (CDC), 172 Jiangsu Rd, Nanjing 210009, China
}

Correspondence should be addressed to Zuhong Lu, zhlu@seu.edu.cn

Received 11 February 2010; Revised 27 March 2010; Accepted 31 March 2010

Academic Editor: Momiao Xiong

Copyright () 2010 Yuhua Qi et al. This is an open access article distributed under the Creative Commons Attribution License, which permits unrestricted use, distribution, and reproduction in any medium, provided the original work is properly cited.

\begin{abstract}
Adenovirus infection can cause various illnesses depending on the infecting serotype, such as gastroenteritis, conjunctivitis, cystitis, and rash illness, but the infection mechanism is still unknown. MicroRNAs (miRNA) have been reported to play essential roles in cell proliferation, cell differentiation, and pathogenesis of human diseases including viral infections. We analyzed the miRNA expression profiles from adenovirus type 3 (AD3) infected Human laryngeal epithelial (Hep2) cells using a SOLiD deep sequencing. 492 precursor miRNAs were identified in the AD3 infected Hep2 cells, and 540 precursor miRNAs were identified in the control. A total of 44 miRNAs demonstrated high expression and 36 miRNAs showed lower expression in the AD3 infected cells than control. The biogenesis of miRNAs has been analyzed, and some of the SOLiD results were confirmed by Quantitative PCR analysis. The present studies may provide a useful clue for the biological function research into AD3 infection.
\end{abstract}

\section{Introduction}

Adenovirus (AD) belongs to a family of nonenveloped icosahedral viruses containing a double-stranded DNA genome, Adenoviridae, and genus Mastadenovirus. There are 53 human AD serotypes divided into seven subgroups (A to $\mathrm{G}$ ) based on immunologic, biologic, and biochemical characteristics [1]. Among these, viruses in groups B1, $\mathrm{C}$, and $\mathrm{E}$ cause respiratory infections, group B2 viruses infect the kidney and urinary tract; group $\mathrm{F}$ viruses cause gastroenteritis; and several group D serotypes are associated with epidemic keratoconjunctivitis [1]. Most people recover from $\mathrm{AD}$ infections by themselves, but people with immunodeficiency and small children are at higher risk of fatal adenovirus infection. Studies showed that AD can evade host immune responses, such as inhibition of interferon functions by RNA and E1A, and inhibition of intrinsic cellular apoptosis in infected cells [2]. The interaction between the virus and its host is still not clear. Recently, several studies evaluated human adenoviruses which play an important role in the study of tumorigenesis, including oncogenic transformation of cells, immune evasion, angiogenesis, and metastasis [3, 4]. There are no antiviral drugs to treat adenoviral infections, so treatment is largely directed at the symptoms. The discovery of the AD yielded further evidence that viruses can sometimes interfere with each other during the growth and replication within a host animal. Other researchers found that this interference can be mediated by interferon produced by the host animal. So interferon has become prominent in the treatment of a variety of cancers and infectious diseases, such as hepatitis C [5]. Recently, several studies demonstrated that RNA interference (RNAi) can also be used for treatment with viral infection in vitro, such as hepatitis B virus, coxsackievirus B3, and Coxsackievirus A16 [6-8]. These findings indicate that those specific siRNAs targeted viruses are not as effective as expected.

RNAi can also be triggered by microRNAs (miRNAs), which are short $\sim 22$ nucleotide RNA sequences that bind complementary sequences in the $3^{\prime}$ UTR of multiple target 
mRNAs, playing an important role in the development, proliferation, differentiation and apoptosis of organisms. Growing evidences have indicated a strong association between microRNAs and various viral infections. For example, miR-125b, mirR-150, miR-382, and miR-223 have been reported to contribute to the maintenance of human immunodeficiency virus-1 (HIV-1) latency in resting CD4 ${ }^{+}$ $\mathrm{T}$ cells [9]. Epstein-Barr virus and Kaposi sarcoma herpes viruses (KSHVs) have been reported to encode miRNAs [10], but the functions of most of them are not known. miRNAs have been reported to be used as a tool for gene specific therapeutics against viral infections [11]. The potential use of miRNAs as novel noninvasive biomarkers for diagnosis has been suggested in other studies [12-14].

In this study, we used a strategy of initial screening by Applied Biosystems' next generation sequencing system which is Sequencing by Oligonucleotide Ligation and Detection (SOLiD) and validation by quantitative RT-PCR (qRTPCR), to analyze the different miRNA expression profiling in AD3 infected Hep2 cells.

\section{Materials and Methods}

2.1. Cell Culture and Virus Infection. Human laryngeal epithelial (Hep2) cells were cultivated in complete RPMI medium 1640 (Invitrogen) supplemented with $10 \%$ fetal calf serum (FCS) at $37^{\circ} \mathrm{C}$ in an atmosphere of $5 \% \mathrm{CO}_{2}$. When the Hep2 cells were grown in $25-\mathrm{cm}^{2}$ flasks reached to $70 \%$ confluence, they were infected with $\mathrm{AD} 3$ at a multiplicity of infection (m.o.i.) of 0.4 under $50 \%$ tissue culture infectious doses $\left(\mathrm{TCID}_{50}\right)$, and maintained after infection at $37^{\circ} \mathrm{C}$ in RPMI 1640 medium with 2\% FCS.

2.2. RNA Isolation. Hep2 cells were infected with $\mathrm{AD} 3$ as described above. At 6 hours, 24 hours, 48 hours, and 72 hours post infection (p.i.), RNA samples were prepared from AD3 infected Hep2 cells and controlled Hep2 cells using a mirVana miRNA Isolation Kit (Ambion): $10^{2}-10^{7}$ cultured cells were washed in 1\% PBS for three times, washed cells were mixed with $600 \mu \mathrm{l}$ Lysis/Binding Solution and $1 / 10$ volume of miRNA Homogenate Additive thoroughly by vortexing and incubated on ice for 10 minutes An equal volume of acid/phenol/chloroform (Ambion) was then added to the each aliquot. The solution was centrifuged for 5 minutes at $10,000 \mathrm{~g}$ at room temperature. For miRNA isolation, the extraction was mixed thoroughly with $1 / 3$ volume of $100 \%$ ethanol and passed through a column (Ambion). The flow-through was contained the small RNAs. The filtrate was then mixed thoroughly with $2 / 3$ volume $100 \%$ ethanol and passed through the second column (Ambion). The column was washed following the protocol, and miRNAs were eluted in $100 \mu \mathrm{l}$ of elution buffer $\left(95^{\circ} \mathrm{C}\right)$. For total RNA isolation, the extraction was mixed thoroughly with 1.25 volume of $100 \%$ ethanol and passed through a column (Ambion). The column was washed following the protocol and total RNA were eluted in $100 \mu \mathrm{l}$ of elution buffer $\left(95^{\circ} \mathrm{C}\right)$. A total of $1 \mu \mathrm{l}$ of the eluate was quantified by Nanodrop.
2.3. SOLiD Sequencing and Sequence Analysis. MiRNA samples (100 ng) isolated from AD3 infected Hep2 cells and control Hep2 cells were processed into sequencing libraries using the Small RNA Expression Kit (Applied Biosystems). Briefly, RNA was ligated overnight with the adapters from the kit, reverse transcribed, RNAse H-treated, and PCRamplified before size selection on Agarose gels to contain 16-61 nt of inserted sequences. Libraries were amplified onto beads using emulsion PCR, deposited on slides, and sequenced using the SOLiD v 2 sequencing system (Applied Biosystems) [15] at the State Key Lab of Bioelectronics Laboratory, Southeast University of China. Finally, SOLiD data was first analyzed by SOLiD System Small RNA Analysis Pipeline Tool (RNA2MAP). Firstly, the parameters set for alignments were the maximum length $(18 \mathrm{nt})$ and the tolerance of 3 mismatches when generating initial seeds locations. In the extension step, less than 6 mismatches were allowed in full length mapping. The miRBase sequences (Sanger) of human being was download from miRBase (http://www.mirbase.org/). Acceptable sequences were compared to the miRBase database (release 14.0). To increase signal above noise, we conservatively selected only those alignments corresponding to beads sampled a minimum of 10 times in any of the libraries.

2.4. Quantitative RT-PCR ( $q R T-P C R$ ). To confirm the expression of miRNAs by deep sequencing approach, stemloop quantitative RT-PCR (qRT-PCR) was performed. Briefly, cDNA was synthesized from total RNA by using AMV reverse transcriptase (TaKaRa). The $20 \mu \mathrm{L}$ reactions were incubated for 15 minutes at $16^{\circ} \mathrm{C}, 30$ minutes at $42^{\circ} \mathrm{C}$, and 5 minutes at $85^{\circ} \mathrm{C}$, and then held at $4^{\circ} \mathrm{C}$. Subsequently, the $20 \mu \mathrm{L}$ PCR reactions included $1 \mu \mathrm{l}$ RT-PCR product, $10 \mu \mathrm{l}$ Premix Ex Taq (TaKaRa), and $1 \mu$ l SYBR green (Invitrogen). The reactions were incubated in a 96-well optical plate at $95^{\circ} \mathrm{C}$ for 10 minutes, followed by 40 cycles of $95^{\circ} \mathrm{C}$ for 15 seconds, and $60^{\circ} \mathrm{C}$ for 1 minute. Q-PCR assay was performed in triplicate using an ABI 7500 machine (Applied Biosystems). The expression levels of candidate miRNAs were measured in terms of threshold cycle value $\left(\mathrm{C}_{\mathrm{T}}\right)$ and normalized to Human small nuclear RNA U6. The ratio of two groups of miRNAs was calculated by using the equation $2^{-\Delta \Delta \mathrm{CT}}$ [16], in which $\Delta \mathrm{CT}_{1}$ (infected cells) $=\mathrm{C}_{\mathrm{T} 1}-\mathrm{C}_{\mathrm{TU} 6}$, $\Delta \mathrm{CT}_{2}$ (control cells) $=\mathrm{C}_{\mathrm{T} 2}-\mathrm{C}_{\mathrm{TU} 6}$ and $\Delta \Delta \mathrm{CT}=\Delta \mathrm{CT}_{1}-$ $\Delta \mathrm{CT}_{2}$.

2.5. Statistical Analysis. We firstly used the Z-test to determine the statistical significance of the differences between the two libraries. This approach is to look at the number of copies of a specic miRNA per cell as a fraction or proportion of the total number of miRNA molecules in that cell. The same proportion $(p)$ of specic tags should be present in the miRNA library of all sequenced tags [17]

$$
p=\frac{n_{\text {specific miRNA }} / \text { cell }}{N_{\text {total miRNA }} / \text { cell }}=\frac{n_{\text {specific miRNA }}}{N_{\text {total miRNA }}} .
$$


So the $Z$-value for a specific miRNA could be calculated as following:

$$
Z \text {-value }=\frac{p_{\text {test }}-p_{\text {control }}}{\sqrt{p_{0}\left(1-p_{0}\right) / N_{\text {test }}+p_{0}\left(1-p_{0}\right) / N_{\text {control }}}} .
$$

The proportion $p_{0}$, the expected proportion when the null hypothesis is true, is calculated as $p_{0}=\left(n_{\text {test }}+\right.$ $\left.n_{\text {control }}\right) /\left(N_{\text {test }}+N_{\text {control }}\right)$. The $Z$ statistic is approximately normally distributed and can be compared with critical $Z$ value for the two-sided significance level $\alpha$ [17].

A $Z$-test was used to deal with the raw data by considering both the copies and fold change of miRNAs. Candidate miRNAs should be full three criteria: (1) mean fold change $>2$, (2) having at least 10 copies by SOLiD sequencing, and (3) $Z$ score $>1.96$ or $<-1.96$ for $P<.05$.

\section{Results}

3.1. Replication of AD3 in Hep2 Cells. Cytopathic effect (CPE) was first observed at 24 hours post infection and progressed to moderate and severe CPE at 72 hours and 96 hours, respectively. miRNA extracted from cells after 72 hours of infection was used for SOLiD deep sequencing.

3.2. SOLiD Sequencing of miRNAs from Hep2 Cells Infected by AD3 and Control Hep2 Cells. To search for the miRNAs expression in AD3 infected Hep2 cells and the control, we prepared these two miRNA samples for sequencing by the SOLiD system. 358,297 effective reads were obtained in AD3 infected cells and 479,414 effective reads in control cells. After filtrating reads contaminated by rRNAetc (rRNA, tRNA, snRNA, and snoRNA), repeat fractions, and degraded fractions, the remaining effective reads were mapped to the human precursor libraries. 37,800 and 78,143 reads were obtained from infected and control cells, respectively. In these reads, the most abundant size class was $22 \mathrm{nt}$ in both of AD3 infected Hep2 cells and the control. The percentage was $29.3 \%$ and $34.6 \%$ respectively. Following was $23 \mathrm{nt}(27.7 \%$ in AD3 infected Hep2 cells and $26.3 \%$ in the control) (Figure 1). Compared to the miRBase (14.0), 492 precursor, miRNA out of 734 known precursor miRNAs were identified among the AD3 infected Hep2 cells, and 540 precursor miRNAs were identified among the control Hep2 cells. All of the known miRNAs have been characterized in the Sanger miRBase sequence database. Figure 2 showed the distribution of genomic loci in the chromosome. All of the human chromosome were located in the precursor miRNAs. Chromosomes 19 and X contain more precursor miRNAs (12\%, and $11 \%$, resp.). 314 miRNAs (51\%) were located in introns of protein-coding genes, 231 miRNAs (38\%) were located in intergenic regions, while only 43 miRNAs (7\%) were located in exons of noncoding RNAs or UTR of protein coding genes. Interestedly, 23 miRNAs (4\%) were located in either an exon or an intron depending on alternative splicing of the host transcript (Table 1).

3.3. Expression Profiles of miRNAs in AD3 Infected Cells. To study the differential expression profile of known miRNAs

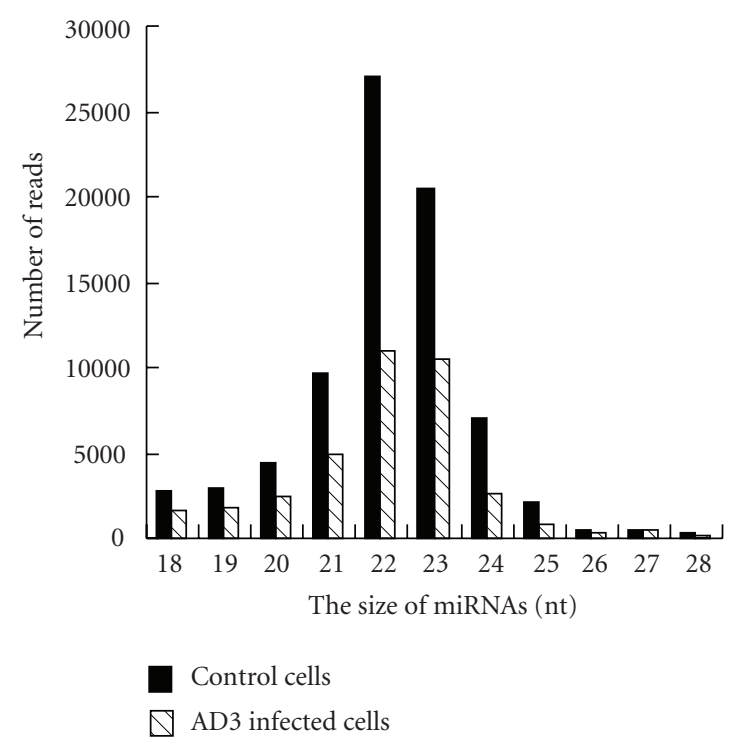

Figure 1: Size distribution of sequenced miRNAs.

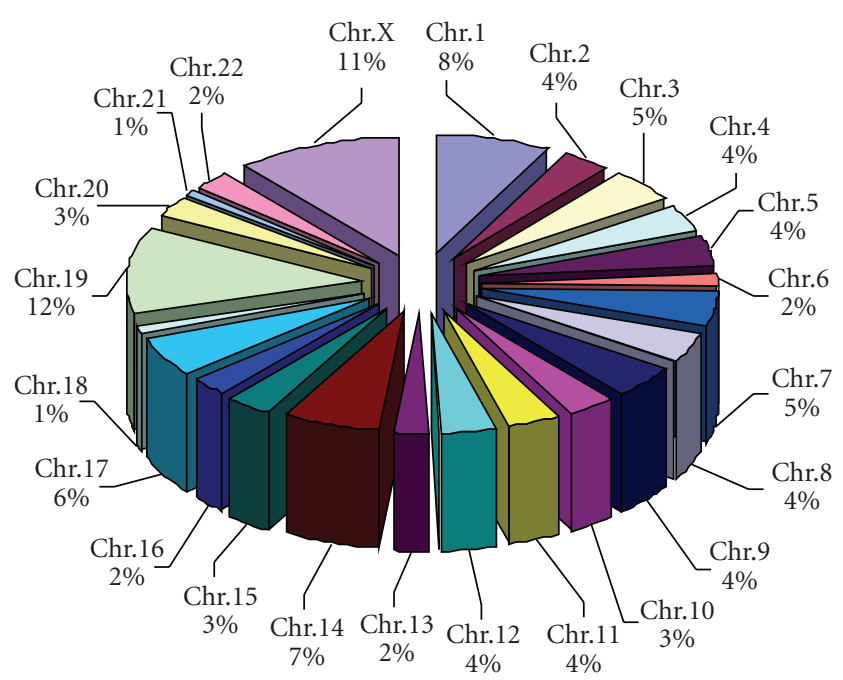

Figure 2: Genomic loci of the sequenced miRNAs (Chr: chromosome).

TABle 1: Transcript loci of sequenced miRNAs.

\begin{tabular}{lcc}
\hline Loci & Number of miRNAs & Percentage (\%) \\
\hline intron & 314 & 51 \\
intergenic & 231 & 38 \\
Exon and Utr & 42 & 7 \\
Exon, intron (mixed) & 23 & 4 \\
\hline
\end{tabular}

between AD3 infected Hep2 cells and the control, both the $Z$-test and fold-change analysis were made based on the sequencing results. There are 80 precursor miRNAs to meet the three criteria described above, and could be selected as candidates. Among these candidates, 44 precursor miRNAs were shown upregulated expression in AD3 infected cells (Table 2). $Z$-test value was from 1.99 to 49.33 . 19 out of 44 
TABLE 2: Upexpressed miRNAs in infected cells compared with noninfected cells.

\begin{tabular}{|c|c|c|c|c|}
\hline miRNA Name & Reads in infected cells & Reads in non-infected cells & $Z$ score & $\begin{array}{c}\text { Ratio of } \\
\text { infected/non-infected } \\
\text { (Normalized })\end{array}$ \\
\hline hsa-miR-1975 & 1937 & 548 & 49.33 & 7.47 \\
\hline hsa-miR-1302-2 & 788 & 228 & 31.13 & 7.31 \\
\hline hsa-miR-191 & 868 & 566 & 23.16 & 3.24 \\
\hline hsa-miR-17 & 1238 & 1105 & 21.65 & 2.37 \\
\hline hsa-miR-92a-2 & 1166 & 1084 & 20.17 & 2.27 \\
\hline hsa-miR-1979 & 444 & 201 & 20.02 & 4.67 \\
\hline hsa-miR-92a-1 & 1184 & 1146 & 19.48 & 2.18 \\
\hline hsa-miR-1302-3 & 197 & 58 & 15.45 & 7.18 \\
\hline hsa-miR-1302-8 & 191 & 54 & 15.38 & 7.48 \\
\hline hsa-miR-1302-6 & 191 & 57 & 15.16 & 7.09 \\
\hline hsa-miR-1302-7 & 191 & 57 & 15.16 & 7.09 \\
\hline hsa-miR-1302-1 & 180 & 59 & 14.32 & 6.45 \\
\hline hsa-miR-1302-5 & 165 & 50 & 14.03 & 6.98 \\
\hline hsa-miR-1302-4 & 161 & 50 & 13.76 & 6.81 \\
\hline hsa-miR-7-3 & 111 & 18 & 13.13 & 13.04 \\
\hline hsa-miR-7-1 & 150 & 50 & 13.00 & 6.34 \\
\hline hsa-miR-7-2 & 109 & 21 & 12.64 & 10.97 \\
\hline hsa-miR-1974 & 262 & 188 & 11.88 & 2.95 \\
\hline hsa-miR-1246 & 186 & 121 & 10.70 & 3.25 \\
\hline hsa-miR-378 & 220 & 199 & 8.95 & 2.34 \\
\hline hsa-miR-18a & 185 & 153 & 8.92 & 2.56 \\
\hline hsa-miR-619 & 79 & 41 & 7.92 & 4.07 \\
\hline hsa-miR-25 & 178 & 172 & 7.52 & 2.19 \\
\hline hsa-miR-421 & 57 & 38 & 5.82 & 3.17 \\
\hline hsa-miR-1273 & 54 & 37 & 5.57 & 3.09 \\
\hline hsa-miR-18b & 62 & 51 & 5.18 & 2.57 \\
\hline hsa-miR-345 & 50 & 37 & 5.07 & 2.86 \\
\hline hsa-miR-342 & 83 & 84 & 4.87 & 2.09 \\
\hline hsa-miR-744 & 28 & 19 & 4.03 & 3.12 \\
\hline hsa-miR-505 & 22 & 13 & 3.90 & 3.58 \\
\hline hsa-miR-1247 & 17 & 10 & 3.43 & 3.59 \\
\hline hsa-miR-942 & 12 & 6 & 3.14 & 4.23 \\
\hline hsa-miR-635 & 11 & 5 & 3.14 & 4.65 \\
\hline hsa-miR-627 & 12 & 7 & 2.90 & 3.62 \\
\hline hsa-miR-150 & 10 & 5 & 2.87 & 4.23 \\
\hline hsa-miR-566 & 10 & 5 & 2.87 & 4.23 \\
\hline hsa-miR-324 & 20 & 18 & 2.71 & 2.35 \\
\hline hsa-miR-146b & 10 & 6 & 2.60 & 3.52 \\
\hline hsa-miR-454 & 10 & 6 & 2.60 & 3.52 \\
\hline hsa-miR-1972 & 14 & 12 & 2.37 & 2.47 \\
\hline hsa-miR-320c-2 & 14 & 13 & 2.20 & 2.28 \\
\hline hsa-miR-589 & 16 & 16 & 2.17 & 2.11 \\
\hline
\end{tabular}

upregulated precursor miRNAs showed higher values (over 10). Among this, miR-1975 and miR-1302-2 have the highest $Z$ values with 49.33 and 31.13, respectively. Table 2 showed the ratio of miRNAs expression level of AD3 infected cells versus control cells. Among this, miR-1975 and miR-1302-2 have the higher ratio (7.47-fold and 7.31-fold). 36 precursor miRNAs were shown downregulated expression in AD3 infected cells (Table 3). Among this there are 11 miRNAs that have the higher $Z$ values (over 10). Table 3 showed the ratio of AD3 infected cells/control cells (expression level 
TABLE 3: Downexpressed miRNAs in infected cells compared with noninfected cells.

\begin{tabular}{|c|c|c|c|c|}
\hline miRNA Name & Reads in infected cells & Reads in non-infected cells & $Z$ score & $\begin{array}{c}\text { Ratio of } \\
\text { infected/non-infected } \\
\text { (Normalized })\end{array}$ \\
\hline hsa-miR-1184 & 3 & 21 & -2.06 & 0.30 \\
\hline hsa-miR-1180 & 10 & 43 & -2.06 & 0.49 \\
\hline hsa-miR-219-1 & 8 & 38 & -2.14 & 0.45 \\
\hline hsa-miR-519a-1 & 6 & 33 & -2.24 & 0.38 \\
\hline hsa-miR-433 & 6 & 35 & -2.40 & 0.36 \\
\hline hsa-miR-650 & 6 & 35 & -2.40 & 0.36 \\
\hline hsa-miR-148b & 12 & 55 & -2.49 & 0.46 \\
\hline hsa-miR-148a & 13 & 60 & -2.62 & 0.46 \\
\hline hsa-miR-196b & 4 & 31 & -2.62 & 0.27 \\
\hline hsa-miR-1259 & 6 & 46 & -3.18 & 0.28 \\
\hline hsa-miR-362 & 30 & 128 & -3.53 & 0.50 \\
\hline hsa-miR-181b-1 & 7 & 60 & -3.80 & 0.25 \\
\hline hsa-miR-374b & 8 & 65 & -3.87 & 0.26 \\
\hline hsa-miR-655 & 26 & 126 & -3.96 & 0.44 \\
\hline hsa-miR-33a & 16 & 95 & -3.99 & 0.36 \\
\hline hsa-miR-101-1 & 44 & 191 & -4.40 & 0.49 \\
\hline hsa-miR-101-2 & 44 & 192 & -4.43 & 0.48 \\
\hline hsa-miR-452 & 29 & 158 & -4.87 & 0.39 \\
\hline hsa-miR-181b-2 & 8 & 91 & -5.12 & 0.19 \\
\hline hsa-miR-26b & 31 & 172 & -5.14 & 0.38 \\
\hline hsa-let-7e & 33 & 199 & -5.84 & 0.35 \\
\hline hsa-miR-1274b & 15 & 168 & -6.93 & 0.19 \\
\hline hsa-miR-27b & 139 & 677 & -9.25 & 0.43 \\
\hline hsa-miR-34a & 206 & 904 & -9.70 & 0.48 \\
\hline hsa-miR-582 & 82 & 531 & -9.95 & 0.33 \\
\hline hsa-miR-338 & 113 & 905 & -14.40 & 0.26 \\
\hline hsa-miR-27a & 248 & 1382 & -14.68 & 0.38 \\
\hline hsa-miR-125b-1 & 399 & 2082 & -17.23 & 0.41 \\
\hline hsa-miR-125b-2 & 443 & 2220 & -17.25 & 0.42 \\
\hline hsa-miR-30b & 219 & 1572 & -18.12 & 0.29 \\
\hline hsa-miR-30c-2 & 233 & 1812 & -20.19 & 0.27 \\
\hline hsa-miR-30c-1 & 232 & 1811 & -20.22 & 0.27 \\
\hline hsa-miR-210 & 207 & 1738 & -20.40 & 0.25 \\
\hline hsa-miR-19b-1 & 538 & 2952 & -21.36 & 0.39 \\
\hline hsa-miR-19b-2 & 536 & 2946 & -21.36 & 0.38 \\
\hline hsa-miR-30a & 260 & 2083 & -21.95 & 0.26 \\
\hline
\end{tabular}

for precursor miRNAs). Among this miR-181b-2 and miR$1274 \mathrm{~b}$ showed lowest change in AD3 infected cells (0.19 fold) compared with control cells.

3.4. Q-PCR Detection for miRNAs Profiles in AD3 Infected Cells. To confirm the differential expression of miRNAs in AD3 infected Hep2 cells, we performed some miRNAs tests with stem-loop Q-PCR assays in total RNA isolated from AD3 infected Hep2 cells and the control, respectively. The expression levels of candidate miRNAs were measured in terms of threshold cycle value $\left(\mathrm{C}_{\mathrm{T}}\right)$ and normalized to Human small nuclear RNA U6. The ratio of miRNAs in two groups was calculated by using the equation $2^{-\Delta \Delta \mathrm{CT}}$. The results showed that there was general consistency between Q-PCR assay and SOLiD sequencing analysis. The seven upregulated miRNAs in SOLiD results showed increased ratios measured in Q-PCR assays (Figure 3). For example, miR-1974, miR-1975, and miR-7 showed increasing values of 2.51, 4.87 and 2.44 respectively measured in Q-PCR assay, and increasing values of $2.95,7.47$ and 6.34 were obtained in SOLiD sequencing (Table 2). The seven downregulated 


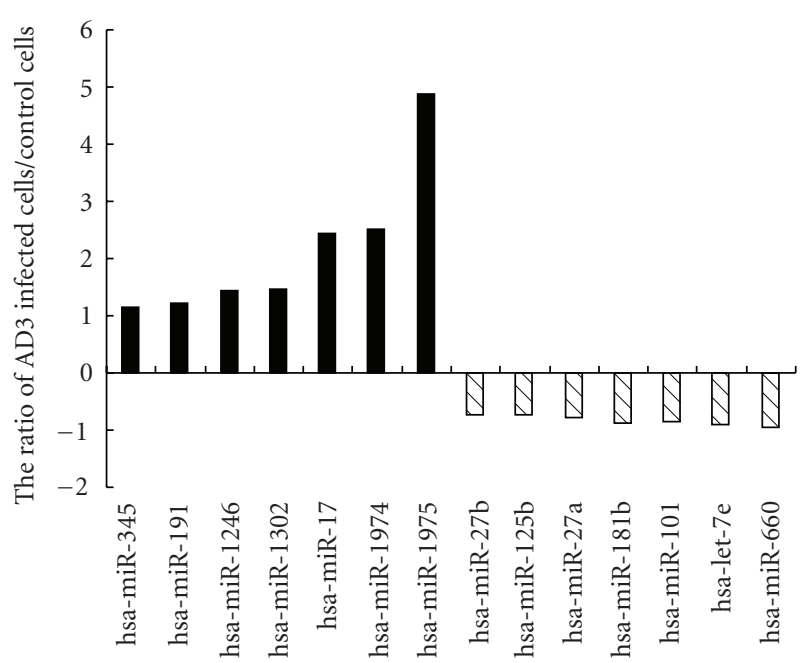

FIGURE 3: Quantitation of miRNA expression levels in the AD3 infected Hep 2 cells and controls. Y values are the ratio of two groups of miRNAs calculated by using the equation $2^{-\Delta \Delta C T}$. The black column is for upregulated miRNAs, and the gray for downregulated miRNAs.

miRNAs in SOLiD results showed decrease ratios measured in Q-PCR assays (Figure 3). For example, the results showed decreased expression levels in miR-27b, miR-125b, and miR27a in AD3 infected Hep2 cells with fold change of 0.74, 0.75 , and 0.77 respectively (Figure 3 ). While in SOLiD sequencing analysis, these miRNAs have shown decreased expression with fold change $0.43,0.41$ and 0.38 , respectively (Table 3). To further understand the miRNAs expression in AD3 infected Hep2 cells at 6 hours, 24 hours, 48 hours, and 72 hours post infection, we performed Q-PCR experiments on miRNAs chosen from Figure 3. The ratio of miRNAs in AD3 infected cells versus controls was calculated by using the equation $2^{-\Delta \Delta C T}$. Surprisingly, all of the miRNAs shown in Table 4 exhibited increased expression in AD3 infected cells at 6 hours post infection. However, at 48 hours p.i. and 72 hours p.i., miR-27b, miR-125b, and miR-181b showed downregulated expression in AD3 infected cells comparing controls (Table 4). At 48 hours p.i. and 72 hours p.i., miR1974 showed increased expression in AD3 infected cells with fold change 1.36 and 1.26 respectively. However, at 24 hours p.i., miR-1974 showed decreased expression in AD3 infected cells. Table 4 showed that the miR-17 expression was upregulated at 6, 24, and 72 hours p.i. in AD3 infected cells. But at 48 hours p.i., miR-17 showed downregulated expression in AD3 infected cells.

\section{Discussion}

As second-generation sequencing platforms have matured, all three major high-throughput sequencing systemsIllumina's Genome Analyzer (Solexa), Life Technologies' ABI SOLiD, and Roche's 454 GS FLX have been used to study miRNA expression profiles associated with disease such as cancer, viral and metabolic diseases. Compared with the microarray technology, deep sequencing can generate
TABLE 4: The ratio of ADV-3 infected cells/control cells at different time course after the infection.

\begin{tabular}{lcccc}
\hline miRNAs & $\begin{array}{c}6 \text { hours } \\
\text { p.i. }\end{array}$ & $\begin{array}{c}24 \text { hours } \\
\text { p.i. }\end{array}$ & $\begin{array}{c}48 \text { hours } \\
\text { p.i. }\end{array}$ & $\begin{array}{c}72 \text { hours } \\
\text { p.i. }\end{array}$ \\
\hline hsa-miR-27b & 2.77 & 1.25 & 0.32 & 0.66 \\
hsa-miR-125b & 2.39 & 0.47 & 0.56 & 0.84 \\
hsa-miR-181b & 1.73 & 0.62 & 0.52 & 0.62 \\
hsa-miR-1974 & 1.62 & 0.4 & 1.36 & 1.26 \\
hsa-miR-17 & 2.81 & 1.6 & 0.44 & 1.33 \\
\hline
\end{tabular}

p.i. refers to post infection. The ratio of miRNAs in two groups was calculated by using the equation $2^{-\Delta \Delta C T}$.

millions of small RNA sequence reads from a given sample, giving possible to discover the novel miRNAs or study the function of virus encoded miRNAs by using bioinformatics and molecular biology tools. miRNAs have recently been recognized as major regulators of gene expression in various viral infections. Virus-encoded miRNAs seem to evolve rapidly and regulate both the viral life cycle and the interaction between viruses and their hosts. In the present study, we have screened out some miRNA candidates that have obvious differential expression from AD3 infected Hep2 cells by SOLiD deep sequencing system.

This study identified 492 precursor miRNAs in the AD3 infected cells and more precursor miRNAs in control cells by mapping the human miRBase database. Out of these almost $38 \%$ were located in intergenic regions, which usually contain their own miRNA gene promoter and regulatory units [18]. However, as much as $51 \%$ miRNAs were located in introns, and only $7 \%$ were located in exons of noncoding RNAs or UTR of protein coding genes. These usually show a concurrent transcription and regulation expression profile originating from a common promoter with their host genes [19-21]. Some pre-miRNAs existing in drosphila and $c$ elegants have been reported to be spliced from the introns in which they reside without having to undergo the microprocessor machinery. These miRNAs are called mirtrons $[22,23]$. Since our study identified $51 \%$ miRNAs were located in introns, we think more mirtons could be discovered through bioinformatics and molecular biology tools.

The interaction between the $\mathrm{AD}$ and its host is still unknown. One of the mechanisms is that the virus must interfere with the host cell antiviral defense mechanisms to maximize escape and spread of the progeny virus during a virus infection [24]. Previous studies determine the changes in the host cell gene expression profiles upon infection with AD using DNA microarray technique [24, 25]. For example, many transcriptions factor E2F-dependent host cell genes showed different expression during AD infection. 45\% of the upregulated genes and $25 \%$ of the downregulated genes contained potential E2F binding sites [24]. It is showen that E2F-dependent host cell genes are subjected to a selective regulation. However, the mechanism of $\mathrm{AD}$ infection after transcription is still unknown. miRNA can regulate gene expression through translational repression or mRNA cleavage. Previous research has reported that the 
expression of E2F1 is negatively regulated by two c-mycregulated miRNAs, miR-17-5p, and miR-20a [26]. In our study, upregulated miR-17 may target with transcription factor E2F1 [26], playing important role in cell differentiation, proliferation, and apoptosis. Out of these, miR-17 has been reported to be overexpression in B-cell lymphoma samples [27]. The high miR-17 cluster miRNAs suppress cell death [27]. This suggests that during a virus infection, cellular apoptosis must delay long enough and the virus needs to establish optimal conditions for replication to ensure efficient production of progeny virus. Our study may provide a useful clue for the biological function research into AD3 infected host cells. Further investigation needs to clarify the roles of identified miRNAs in the pathogenesis of $\mathrm{AD} 3$ infected host cells.

\section{Conclusion}

SOLiD sequencing provides a useful method for identification of the miRNAs profiles in AD3 infected Hep2 cells. 492 precursor miRNAs were identified in the AD3 infected Hep2 cells, and 540 precursor miRNAs were identified in the control. A total of 44 miRNAs demonstrated high expression and 36 miRNAs showed lower expression in the AD3 infected cells than the control. Further studies are required to identify the miRNA target genes and the functions of the miRNAs in the complex molecular network regulation during the virus infection host cells using bioinformatics tools.

\section{Acknowledgments}

The paper was supported by the National Natural Science Foundation of China (30901285), the Natural Science Foundation of Jiangsu Province of China (SBK200922783), and the Open Research Fund of State Key Laboratory of Bioelectronics, Southeast University of China. Both Yuhua Qi and Jin Tu contributed equally to this work.

\section{References}

[1] M. Echavarría, "Adenoviruses in immunocompromised hosts," Clinical Microbiology Reviews, vol. 21, no. 4, pp. 704-715, 2008.

[2] J. A. Mahr and L. R. Gooding, "Immune evasion by adenoviruses," Immunological Reviews, vol. 168, pp. 121-130, 1999.

[3] H. Guan, J. F. Williams, and R. P. Ricciardi, "Induction of neuronal and tumor-related genes by adenovirus type 12 E1A," Journal of Virology, vol. 83, no. 2, pp. 651-661, 2009.

[4] G. Chinnadurai, "Modulation of oncogenic transformation by the human adenovirus E1A C-terminal region," Current Topics in Microbiology and Immunology, vol. 273, pp. 139-161, 2004.

[5] K. A. Sharieff, D. Duncan, and Z. Younossi, "Advances in treatment of chronic hepatitis C: 'pegylated' interferons," Cleveland Clinic Journal of Medicine, vol. 69, no. 2, pp. 155159, 2002.

[6] Z. Wu, Y. Gao, L. Sun, P. Tien, and Q. Jin, "Quick identification of effective small interfering RNAs that inhibit the replication of coxsackievirus A16," Antiviral Research, vol. 80, no. 3, pp. 295-301, 2008.
[7] K.-L. Wu, X. Zhang, J. Zhang, et al., "Inhibition of hepatitis $B$ virus gene expression by single and dual small interfering RNA treatment," Virus Research, vol. 112, no. 1-2, pp. 100107, 2005.

[8] J. Yuan, P. K. M. Cheung, H. M. Zhang, D. Chau, and D. Yang, "Inhibition of coxsackievirus B3 replication by small interfering RNAs requires perfect sequence match in the central region of the viral positive strand," Journal of Virology, vol. 79, no. 4, pp. 2151-2159, 2005.

[9] J. Huang, F. Wang, E. Argyris, et al., "Cellular microRNAs contribute to HIV-1 latency in resting primary $\mathrm{CD}^{+} \mathrm{T}$ lymphocytes," Nature Medicine, vol. 13, no. 10, pp. 1241-1247, 2007.

[10] V. Nair and M. Zavolan, "Virus-encoded microRNAs: novel regulators of gene expression," Trends in Microbiology, vol. 14, no. 4, pp. 169-175, 2006.

[11] S.-Y. Ying and S.-L. Lin, "Current perspectives in intronic micro RNAs (miRNAs)," Journal of Biomedical Science, vol. 13, no. 1, pp. 5-15, 2006.

[12] J. Skog, T. Wurdinger, S. van Rijn, et al., "Glioblastoma microvesicles transport RNA and proteins that promote tumour growth and provide diagnostic biomarkers," Nature Cell Biology, vol. 10, no. 12, pp. 1470-1476, 2008.

[13] K. E. Resnick, H. Alder, J. P. Hagan, D. L. Richardson, C. M. Croce, and D. E. Cohn, "The detection of differentially expressed microRNAs from the serum of ovarian cancer patients using a novel real-time PCR platform," Gynecologic Oncology, vol. 112, no. 1, pp. 55-59, 2009.

[14] S. Gilad, E. Meiri, Y. Yogev, et al., "Serum microRNAs are promising novel biomarkers," PLoS ONE, vol. 3, no. 9, article e3148, 2008.

[15] L. A. Goff, J. Davila, M. R. Swerdel, et al., "Ago2 immunoprecipitation identifies predicted MicroRNAs in human embryonic stem cells and neural precursors," PLoS ONE, vol. 4, no. 9, article e7192, 2009.

[16] T. D. Schmittgen and K. J. Livak, "Analyzing real-time PCR data by the comparative CT method," Nature Protocols, vol. 3, no. 6, pp. 1101-1108, 2008.

[17] A. J. Kal, A. J. van Zonneveld, V. Benes, et al., "Dynamics of gene expression revealed by comparison of serial analysis of gene expression transcript profiles from yeast grown on two different carbon sources," Molecular Biology of the Cell, vol. 10, no. 6, pp. 1859-1872, 1999.

[18] N. C. Lau, L. P. Lim, E. G. Weinstein, and D. P. Bartel, "An abundant class of tiny RNAs with probable regulatory roles in Caenorhabditis elegans," Science, vol. 294, no. 5543, pp. 858862, 2001.

[19] Y.-K. Kim and V. N. Kim, "Processing of intronic microRNAs," The EMBO Journal, vol. 26, no. 3, pp. 775-783, 2007.

[20] S. Baskerville and D. P. Bartel, "Microarray profiling of microRNAs reveals frequent coexpression with neighboring miRNAs and host genes," RNA, vol. 11, no. 3, pp. 241-247, 2005.

[21] A. Rodriguez, S. Griffiths-Jones, J. L. Ashurst, and A. Bradley, "Identification of mammalian microRNA host genes and transcription units," Genome Research, vol. 14, no. 10, pp. 1902-1910, 2004.

[22] K. Okamura, J. W. Hagen, H. Duan, D. M. Tyler, and E. C. Lai, "The mirtron pathway generates microRNA-class regulatory RNAs in Drosophila," Cell, vol. 130, no. 1, pp. 89-100, 2007.

[23] J. G. Ruby, C. H. Jan, and D. P. Bartel, "Intronic microRNA precursors that bypass Drosha processing," Nature, vol. 448, no. 7149 , pp. 83-86, 2007. 
[24] H. Zhao, F. Granberg, L. Elfineh, U. Pettersson, and C. Svensson, "Strategic attack on host cell gene expression during adenovirus infection," Journal of Virology, vol. 77, no. 20, pp. 11006-11015, 2003.

[25] A. Dorn, H. Zhao, F. Granberg, et al., "Identification of specific cellular genes up-regulated late in adenovirus type 12 infection," Journal of Virology, vol. 79, no. 4, pp. 2404-2412, 2005.

[26] K. A. O’Donnell, E. A. Wentzel, K. I. Zeller, C. V. Dang, and J. T. Mendell, "c-Myc-regulated microRNAs modulate E2F1 expression,” Nature, vol. 435, no. 7043, pp. 839-843, 2005.

[27] L. He, J. M. Thomson, M. T. Hemann, et al., "A microRNA polycistron as a potential human oncogene," Nature, vol. 435, no. 7043, pp. 828-833, 2005. 

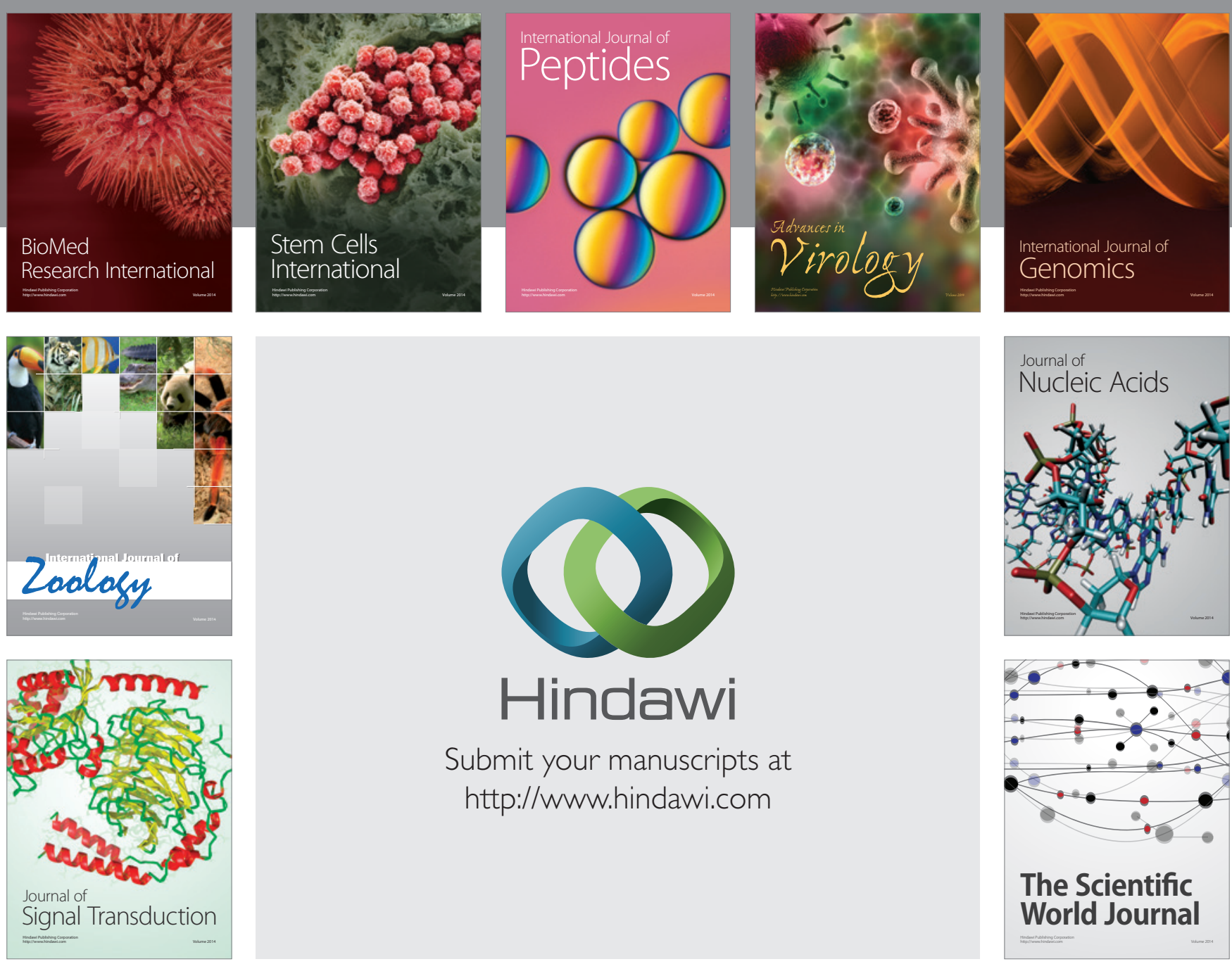

Submit your manuscripts at

http://www.hindawi.com
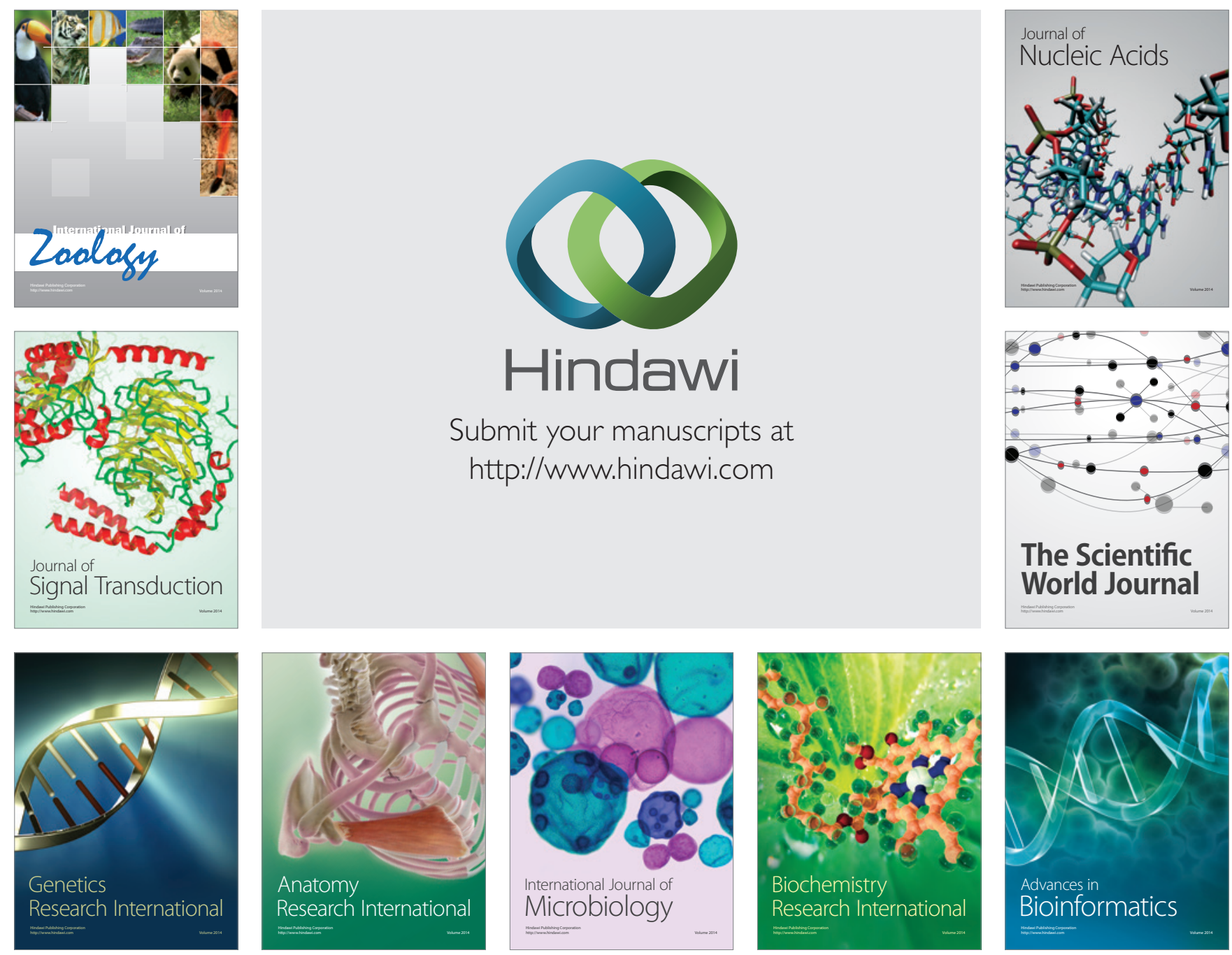

The Scientific World Journal
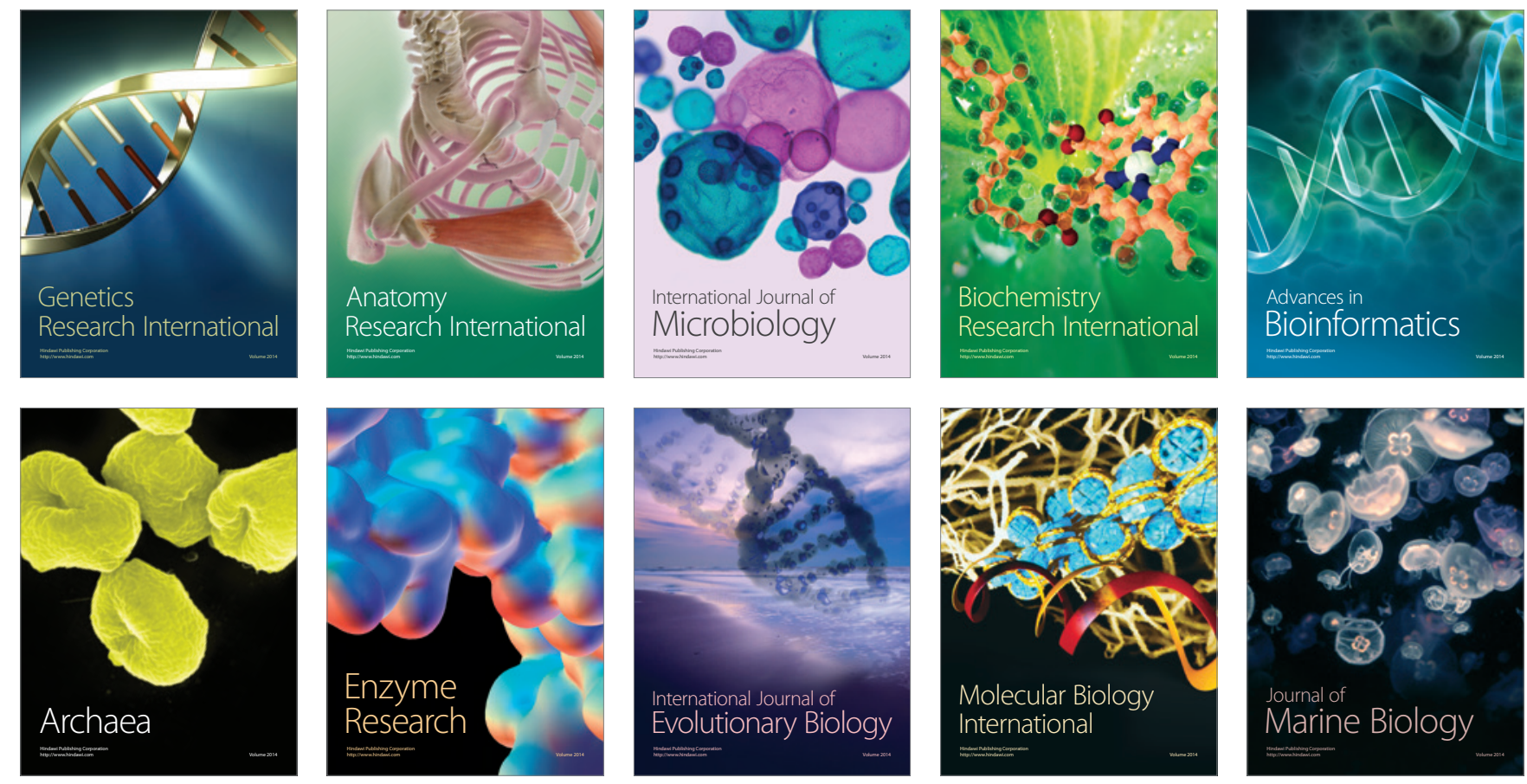